Revista Brasil. Bot., V.25, n.3, p.323-329, set. 2002

\title{
Estrutura fitossociológica da comunidade de lianas em uma floresta estacional semidecidual na Fazenda Canchim, São Carlos, SP
}

\author{
REGIS C. HORA ${ }^{2,3}$ e JOÃO J. SOARES ${ }^{2}$
}

(recebido: 13 de março de 2002; aceito: 22 de maio de 2002)

\begin{abstract}
Phytosociological structure of a liana community in a seasonal semideciduous forest at Fazenda Canchim, São Carlos, SP). A phytosociological research concerning liana vegetation has been accomplished at Fazenda Canchim Embrapa/CPPSE. The forest under analysis, which is fragmented in two parts, covers an area of 112 ha, located at $21^{\circ} 57^{\prime} \mathrm{S}$ and $47^{\circ} 50^{\prime} \mathrm{W}$. 75 plots of $10 \times 10 \mathrm{~m}$ were studied where all liana individuals had at least $2.5 \mathrm{~cm}$ in diameter, measured $1.3 \mathrm{~m}$ above soil level. Five hundred and twenty eight individuals were found, all of which belonged to 45 species, representing a specific diversity (H') 3.20 nats. Most of the species were among the families Bignoniaceae, Malpighiaceae, Sapindaceae, and Apocynaceae, representing together $71.11 \%$ of all samples. The species with higher importance value (VI) was Mansoa difficilis (Cham.) Bureau \& K. Schum. Most of the sampled individuals were small in diameter and four reached over $10 \mathrm{~cm}$.

RESUMO - (Estrutura fitossociológica da comunidade de lianas em uma floresta estacional semidecidual na Fazenda Canchim, São Carlos, SP). Foi realizado um estudo fitossociológico de lianas em uma floresta estacional semidecidual na Fazenda Canchim - Embrapa/CPPSE. A floresta formada por dois fragmentos, possui uma área total de 112 ha, localizada a $21^{\circ} 57^{\prime} \mathrm{S}$ e 47 $50^{\prime}$ 'W. Foram amostrados em 75 parcelas de $10 \times 10 \mathrm{~m}$ todos os indivíduos de lianas que apresentavam diâmetro maior ou igual a $2,5 \mathrm{~cm}$ a uma distância de $1,3 \mathrm{~m}$ a partir da base do caule fixo no solo. Foram encontrados 528 indivíduos, pertencentes a 45 espécies, o que representou uma diversidade específica (H') de 3,20 nats. Bignoniaceae, Malpighiaceae, Sapindaceae e Apocynaceae foram as famílias com maior número de espécies e, juntas, representaram 71,11\% do total amostrado. A espécie com maior valor de importância (VI) foi Mansoa difficilis (Cham.) Bureau \& K. Schum. Os indivíduos amostrados apresentaram pequenos diâmetros e somente quatro tiveram diâmetros maiores que $10 \mathrm{~cm}$.
\end{abstract}

Key words - Phytosociological structure, lianas, semidecidual forest

\section{Introdução}

As lianas são componentes estruturais importantes e representam parte significativa da flora de florestas tropicais (Putz 1984, Gentry 1982). Nas florestas estacionais semidecíduas do Estado de São Paulo, que sofreram diferentes formas de perturbações e que atualmente encontram-se restritas a pequenos fragmentos (Leitão Filho 1987), as lianas tornaram-se muitas vezes abundantes, podendo, até mesmo, interferir na dinâmica natural destas florestas, muito embora sua presença seja mais benéfica que prejudicial (Morellato \& Leitão Filho 1996; Engel et al. 1998). Alguns fatores que passaram a atuar nestes fragmentos estão relacionados ao efeito de borda, maior incidência de luz, formação de clareiras e maior disponibilidade de suportes, e são favoráveis à ocupação por lianas (Hergaty \& Caballé 1991). Contudo, as evidências da

1. Parte da dissertação de mestrado de R.C. Hora.

2. Universidade Federal de São Carlos, Programa de Pós Graduação em Ecologia e Recursos Naturais, Caixa Postal 676, 13565-905 São Carlos, SP, Brasil.

3. Autor para correspondência: reghora@hotmail.com relação entre lianas e árvores apontam para uma "convivência harmoniosa" entre as espécies, pois somente passam por indesejáveis quando os níveis de distúrbios, na maioria antrópicos, afetam a estrutura e função das comunidades onde estão presentes (Engel et al. 1998).

As lianas, que apresentam rápido crescimento comparado com as espécies arbóreas (Putz 1984), chegam a dominar as copas das árvores suportes e das árvores vizinhas. Para o estudo da dinâmica e manejo florestal, é fundamental o conhecimento deste grupo de vegetais, devido à sua diversidade e importância ecológica (Putz 1984).

Os estudos sobre a distribuição e estrutura das espécies florestais têm se focado, na maioria das vezes, nas espécies arbóreas e/ou arbustivas. Tais estudos contribuem para caracterizar a vegetação como um todo e através destas análises obtêm-se como principais resultados o conhecimento da composição em espécies, organização, ecologia e classificação das comunidades.

No Brasil, são poucos os trabalhos que utilizam as lianas como material de estudo, sendo a ecologia deste grupo, ainda menos conhecida. Alguns trabalhos que contribuem com informações sobre as lianas são: Maia (1990), em floresta de terra firme (Manaus, AM); 
Bernacci \& Leitão Filho (1996), em floresta mesófila semidecídua (Campinas, SP); Kim (1996), em mata atlântica (São Paulo); Lima et al. (1997), em mata atlântica (Rio de Janeiro, RJ); Citadini-Zanette et al. (1997), em mata atlântica (Orleans, SC); Rezende (1997) e Morellato \& Leitão Filho (1998), ambos em florestas estacionais semideciduais no interior do Estado de São Paulo, além de Venturi (2000), em floresta costeira (Viamão, RS). A escassez de estudos sobre as lianas em florestas brasileiras é evidente, sendo necessário, uma maior investigação deste grupo, quer seja sobre sua florística ou estrutura em comunidades vegetais.

O presente trabalho teve como objetivo descrever a estrutura fitossociológica das lianas de uma floresta estacional semidecidual, visando um maior conhecimento sobre alguns aspectos relacionados à ecologia destas plantas.

\section{Material e métodos}

Área de estudo - Este estudo foi realizado em uma Reserva Legal de floresta estacional semidecidual da Fazenda Canchim, Embrapa/CPPSE. A floresta está dividida em dois fragmentos que, juntos, apresentam uma área total de 112 ha. Está localizada a $21^{\circ} 57^{\prime} \mathrm{S}$ e $47^{\circ} 50^{\prime}$ 'W no município de São Carlos, SP, a uma altitude entre 835 e 885 m (figura 1). O clima da região é classificado como Cwa-Awa segundo Köppen, estabelecendo duas estações bem definidas: uma seca, de abril a setembro e outra chuvosa, de outubro a março (Tolentino 1967). O estudo foi realizado no final do ano de 1997 e predominantemente durante o ano de 1998. Durante este período a temperatura média dos meses foi de $25,4^{\circ} \mathrm{C}$ e a precipitação de $1.429 \mathrm{~mm}$. Estes dados foram obtidos na estação meteorológica da Embrapa, localizada a menos de $200 \mathrm{~m}$ da floresta. Sob a floresta estão presentes quatro tipos de solos: Latossolo Vermelho-Amarelo (LV), Terra Roxa Estruturada, Latossolo Roxo (LR) e Latossolo VermelhoEscuro (LE), sendo que este último aparece sob a maioria das unidades amostrais. A textura dos solos é média, com 15 a $34 \%$ de argila e são considerados distróficos, apresentando, todavia, algumas manchas de solos eutróficos (Calderano Filho et al. 1998).

Para o estudo da estrutura fitossociológica das lianas, adotou-se o método de parcelas (Müller-Dombois \& Ellenberg 1974). Foram demarcadas 75 unidades amostrais de $10 \times 10 \mathrm{~m}$, somando uma área total de $7.500 \mathrm{~m}^{2}$. Estas unidades foram instaladas ao longo de transecções perpendiculares a uma trilha que passa pelo fragmento mais conservado de modo que ficaram à direita e à esquerda da trilha. As transecções distaram cerca de $50 \mathrm{~m}$ da borda da floresta e as parcelas, cerca de $20 \mathrm{~m}$ da trilha, desta maneira procurou-se evitar a vegetação marginal exterior do

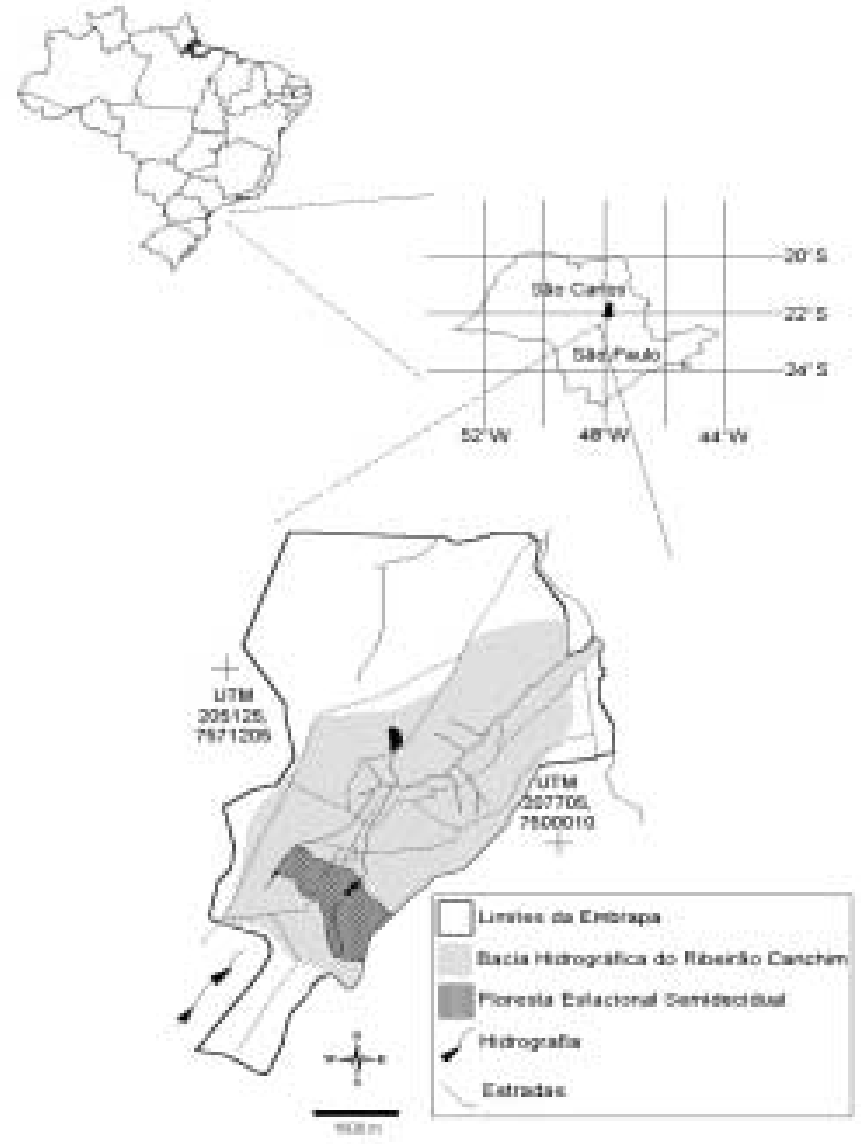

Figura 1. Localização da área de estudo, Reserva de floresta estacional semidecidual da Fazenda Canchim - Embrapa/ CPPSE, São Carlos, SP. Fonte: Embrapa/CPPSE. Organização: C. Henke-Oliveira.

fragmento e também da parte interior, ao longo da trilha. Manteve-se uma distância de $50 \mathrm{~m}$ entre as transecções e $10 \mathrm{~m}$ entre as parcelas. No total foram instaladas 10 transecções, onde o número de parcelas variou de 6 a 10 de cada lado da trilha. Foi amostrado todo indivíduo de liana enraizado, que apresentava brotamento dentro da parcela, com diâmetro do caule igual ou superior a $2,5 \mathrm{~cm}$, medido a 1,3 m de distância da base do caule que emergia do solo.

Os indivíduos de lianas que apresentavam caules extensos, os quais cresceram apoiando-se sobre as árvores produzindo folhas e ramos e que tornavam a tocar o solo, emitindo novas raízes e ramos, foram considerados como indivíduos independentes e amostrados novamente (quando os diâmetros apresentavam tamanho de amostragem). Para a medida dos diâmetros utilizou-se um paquímetro de abertura máxima de $123 \mathrm{~mm}$. Para caules com diâmetros acima desta abertura, ou que se apresentavam muito irregulares foi utilizada uma fita métrica graduada em centímetros. Os valores obtidos com a fita métrica foram, posteriormente, transformados em diâmetros. Os indivíduos enraizados sobre os limites das unidades amostrais foram incluídos na amostra, desde que, na medida de amostragem (1,3 m), seu caule ainda 
permanecesse sobre o limite ou interior da parcela. Como os caules das lianas são muito flexíveis e podem enraizar novamente, em muitos casos isto ocorreu dentro desta medida de 1,3 m. As ramificações de um mesmo indivíduo com tamanho igual ou superior a $2,5 \mathrm{~cm}$ foram medidas e acrescentadas à média para o indivíduo correspondente.

Os indivíduos amostrados foram identificados em campo por uma plaqueta de alumínio, com um número respectivo. Amostras dos indivíduos com material vegetativo ou em fase de reprodução foram coletadas para posterior identificação. Todo material coletado com flor ou fruto foi depositado no herbário do Departamento de Botânica da Universidade Federal de São Carlos (HUFSCar).

Foram calculados os seguintes parâmetros fitossociológicos segundo Müller-Dombois \& Ellenberg (1974): densidades absoluta (DA) e relativa (DR); frequiências absoluta (FA) e relativa (FR); dominâncias absoluta (DoA) e relativa (DoR) e valor de importância (VI).

Para análise da heterogeneidade florística da área quanto às espécies de lianas, calculou-se o índice de diversidade de Shannon (Magurran 1988) e de equabilidade (Pielou 1975).

\section{Resultados e Discussão}

Foram amostradas 45 espécies, pertencentes a 36 gêneros e a 13 famílias (tabela 1). As famílias com maior número de espécies em ordem decrescente foram: Bignoniaceae (18), Malpighiaceae (cinco), Sapindaceae (cinco), Apocynaceae (quatro), representando 71,11\% do total encontrado (figura 2).

Foi observado que, pelo grande número de indivíduos amostrados (528 em 0,75 ha), o número de famílias que dominaram esta sinúsia foi relativamente baixo. Tendo em vista a análise de um grupo específico de plantas (lianas), quatro das unidades amostrais não tiveram indivíduos amostrados.

A composição florística encontrada para o levantamento fitossociológico da floresta da Fazenda Canchim revela que as lianas, predominantemente de caule lenhoso, representam menos da metade das espécies encontradas no levantamento florístico geral da área (109), obtido por Hora (1999). Contudo, as famílias mais importantes em número de espécies são as mesmas nos dois levantamentos, florístico e fitossociológico, realizados nesta área e em outros levantamentos florísticos realizados em fragmentos florestais, com semelhante tipo de vegetação como os de Rezende (1997) e Morellato \& Leitão Filho (1998). Segundo Gentry (1991), este padrão é considerado comum para o Neotrópico, quando se refere às lianas.

Mansoa difficilis foi a espécie com maior densidade relativa (DR), tendo sido encontrados 103 indivíduos que corresponderam a $19,51 \%$ do total de indivíduos amostrados (tabela 2). A segunda espécie que apresentou maior número de indivíduos foi Acacia aff. plumosa (51), que corresponde a $9,66 \%$ do total amostrado. Para as espécies com maior frequiência relativa (FR), as posições das duas primeiras espécies não se alteraram: Mansoa difficilis ocupou a primeira colocação com 11,43\% ocorrendo em 36 unidades amostrais, seguida por Acacia aff. plumosa, que ocorreu em 26 unidades, representando 8,25\% do total. As dez primeiras espécies com maior freqüência relativa somam mais da metade do valor encontrado $(51,11 \%)$, um alto percentual que mostra como poucas espécies estão bem distribuídas na área.

A espécie com maior valor de importância (VI), também foi Mansoa difficilis e, em segundo lugar, Acacia aff. plumosa, que assume esta posição graças ao alto valor apresentado em dominância relativa (tabela 2). Tynanthus elegans apresenta o terceiro maior VI, apesar de ter poucos indivíduos amostrados (14), e ser encontrada em somente sete parcelas. Os dados sobre esta espécie revelam que sua boa colocação neste parâmetro está mais diretamente relacionada ao valor encontrado em dominância relativa $(8,80 \%)$. Este valor é quase duas vezes maior que o encontrado para Heteropterys sp. $(4,49 \%)$, que assume a quinta posição em VI, e três vezes maior que o valor encontrado para Clytostoma campanulatum (2,53\%), quarta posição em VI. Isto pode ser explicado pelo fato dos indivíduos de Tynanthus elegans apresentarem grandes diâmetros, fazendo com que o valor de área basal desta espécie seja superior ao das outras duas espécies relacionadas. Clytostoma campanulatum e Heteropterys sp. apresentaram igual número de indivíduos (25), porém, os diâmetros encontrados para estas espécies são menores.

Entre as 10 principais espécies em VI, do levantamento fitossociológico, destacaram-se ainda: Forsteronia cf. pubescens, Stizophyllum perforatum e Melloa quadrivalvis, que contribuiram com valores significativos de densidade relativa, frequiência relativa e dominância relativa, respectivamente (tabela 2).

Quatro espécies tiveram somente um indivíduo amostrado: Strychnos sp., Paragonia pyramidata, Urvillea laevis e Tetrapterys chlorocapa, correspondendo juntas a $8,88 \%$ do total de espécies encontradas (tabela 2). O aparecimento de somente um indivíduo destas espécies dentro do levantamento fitossociológico não significa que sejam raras, pois foram avistados em outros pontos dentro da floresta.

Pyrostegia venusta que é uma espécie comum em bordas de fragmentos como da área amostrada e 
Tabela 1. Relação das famílias, gêneros e espécies de lianas encontradas no estudo fitossociológico da floresta estacional semidecidual da Fazenda Canchim, São Carlos, SP.

Família

APOCYNACEAE

ASTERACEAE

BIGNONIACEAE

CACTACEAE

CAESALPINIACEAE

DILLENIACEAE

FABACEAE

HIPPOCRATEACEAE

LOGANIACEAE

MALPIGHIACEAE

MIMOSACEAE

SAPINDACEAE
Espécie

Condilocarpon isthmicum (Vell.) A. DC.

Forsteronia sp.

Forsteronia cf. pubescens A. DC.

Indeterminada 1

Mikania sp.

Adenocalymna bracteatum (Cham.) DC.

Amphilophium vauthieri DC.

Anemopaegma chamberlaynii (Cham.) Bureau \& K.Schum.

Arrabidaea conjugata (Vell.) Mart.

Arrabidaea mutabilis Bureau \& K. Schum.

Arrabidaea triplinervea (Mart. ex DC.) Baill.

Arrabidaea selloi (Spreng.) Sandwith

Clytostoma campanulatum (Cham.) Bureau \& K. Schum.

Friedericia speciosa Mart.

Lundia obliqua Sond.

Macfadyena unguis cati (Jacq.) A.H. Gentry

Mansoa difficilis (Cham.) Bureau \& K. Schum.

Melloa quadrivalvis (Jacq.) A.H. Gentry

Paragonia pyramidata (Rich.) Bureau

Pithecoctenium crucigerum (L.) A.H. Gentry

Pyrostegia venusta (Ker-Gawl.) Miers

Stizophyllum perforatum Miers

Thynantus elegans (Cham.) Miers

Pereskia aculeata Mill.

Bauhinia aff. microstachya (Raddi) Macbr.

Davilla rugosa Poir.

Dalbergia frutescens (Vell.) Britton

Machaerium dimorphandrum Hoehne

Hippocratea volubilis $\mathrm{L}$.

Pristimera andina Miers

Semialarium paniculatum (Mart. ex Schult.) N. Hallé

Strychnos sp.

Heteropterys pauciflora A. Juss.

Heteropterys sp.

Stigmaphyllon lalandianum A. Juss.

Tetrapterys chlorocarpa A. Juss.

Tetrapterys guilleminiana A. Juss.

Acacia aff. paniculata Willd.

Acacia aff. plumosa Lowe

Serjania caracasana (Jacq.) Willd.

Serjania laruotteana Cambess.

Thinouia mucronata Radlk.

Urvillea laevis Radlk.

Urvillea ulmacea H.B.K.

Trigonia sp. 


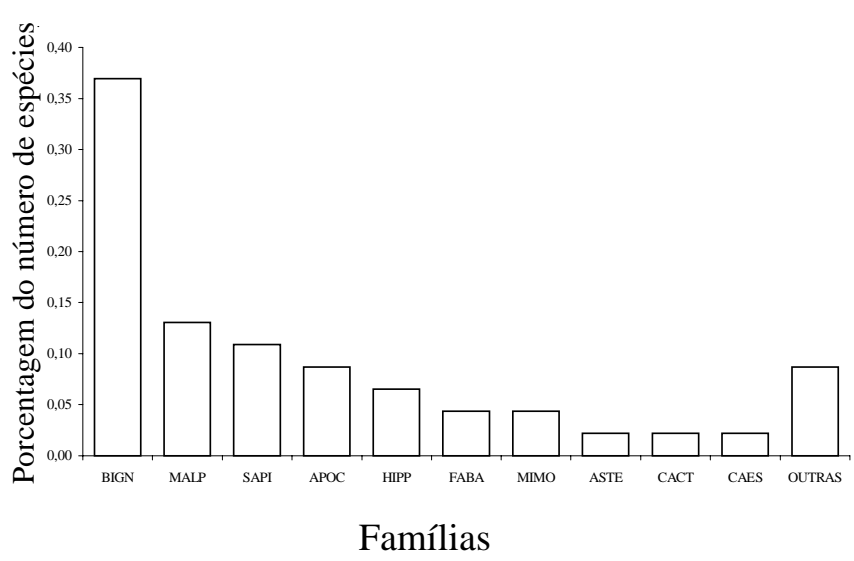

Figura 2. Principais famílias de lianas em porcentagem do número de espécies encontradas na amostragem fitossociológica da floresta estacional semidecidual da Fazenda Canchim, São Carlos, SP. BIGN. Bignoniaceae; MALP. Malpighiaceae; SAPI. Sapindaceae; APOC. Apocynaceae; HIPP. Hippocrateaceae; FABA. Fabaceae; MIMO. Mimosaceae; ASTE. Asteraceae; CACT. Cactaceae; CAES. Caesalpiniaceae.

considerada invasora de culturas e pastagens (Aranha et al. 1982), não apresentou destaque neste levantamento, tendo sido encontrados somente dois indivíduos no interior da floresta com diâmetros acima de $2,5 \mathrm{~cm}$.

A diversidade de lianas em florestas tropicais é um dos principais itens abordados ao se estudar a Flora Neotropical (Putz 1984, Gentry 1991). O índice de diversidade de Shannon (H'), estimado para as espécies de lianas amostradas neste estudo foi de 3,20 nats. Levando em consideração os critérios de inclusão de indivíduos, área amostrada e métodos amostrais, que variam entre os levantamentos fitossociológicos de modo geral, este índice deve ser considerado com cuidado (Oliveira Filho 1989). Uma característica importante para o local de estudo é a existência de uma grande variabilidade de solos em uma área muito pequena. A ocorrência de solos que vão desde álico até eutrófico pode ser um dos fatores responsáveis pela grande diversidade e abundância das lianas. Como são poucos os trabalhos com lianas e nenhum que seja conhecido, pelos autores, em floresta estacional semidecidual com esta abordagem, tornam-se difíceis quaisquer comparações. Citadini-Zanette et al. (1997), encontraram um índice de 1,69 nats, em um remanescente florestal de mata atlântica e consideraram baixa diversidade para a área. Já o valor de equabilidade obtido no presente trabalho, foi de 0,84 o que indica uma dominância ecológica relativamente baixa.

Quanto à análise dos diâmetros dos indivíduos amostrados, observou-se que a maioria pertence às classes de pequenos diâmetros (figura 3). Somente quatro indivíduos apresentaram diâmetros acima de $10 \mathrm{~cm}$. Muitos dos indivíduos amostrados formaram-se de caules que desceram das copas das árvores suportes e, ao tocarem novamente o solo, emitiram novas raízes e ramos, formando novos indivíduos. Este fator é importante quanto à dominância da espécie, pois gera muitos indivíduos que vão pertencer à mesma classe de diâmetro. Em algumas espécies, principalmente em Acacia aff. plumosa, o novo indivíduo formado do caule principal, muitas vezes, torna-se independente.

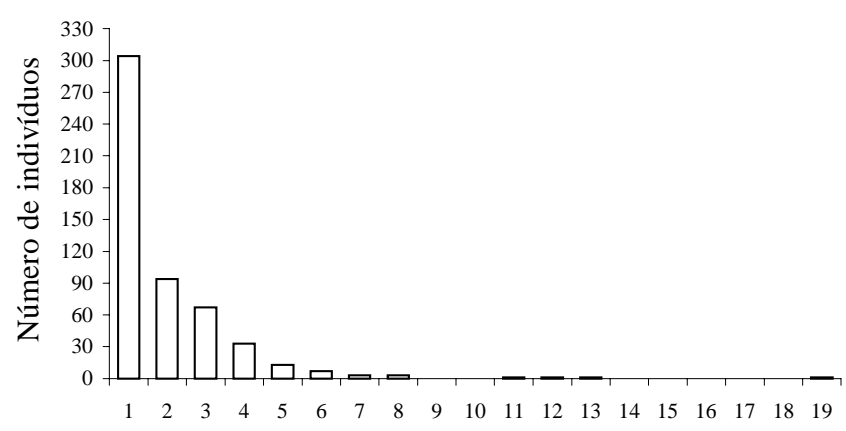

Classe de diâmetro

Figura 3. Freqüência de classes de diâmetro dos indivíduos de lianas encontrados na amostragem fitossociológica da floresta estacional semidecidual da Fazenda Canchim, São Carlos, SP, distribuídos em classes de $1 \mathrm{~cm}(1=2,5$ a $3,5 \mathrm{~cm}$, $2=3,51$ a $4,5 \mathrm{~cm}, \ldots 19=20,51$ a $21,5 \mathrm{~cm})$.

Lianas com diâmetro de caule maior que $10 \mathrm{~cm}$, que poderiam indicar florestas maduras ou antigas (Peixoto \& Gentry 1990), não foram significativas para a área estudada. Levando em conta seu histórico de perturbação com corte seletivo de grandes árvores, entre outras alterações, seriam necessários maiores estudos para embasar este tipo de consideração quanto ao estágio do fragmento estudado, uma vez que as lianas tendem a apresentar crescimento lento em diâmetro (Putz 1990, Hegarty \& Caballé 1991, Teramura et al. 1991), e classes de diâmetros não devem ser utilizados para indicar a idade estrutural de uma população de lianas se comparada com árvores (Hegarty \& Caballé 1991), pois as lianas investem mais no crescimento em extensão (Putz 1990), dificultando uma estimativa da sua fase de desenvolvimento.

Com a análise dos resultados obtidos, evidenciase uma alta diversidade de lianas na área estudada. A heterogeneidade de habitats existentes, juntamente com os eventos antrópicos do passado e biológicos que fazem parte da história da floresta, contribuem para a 
Tabela 2. Relação das espécies de lianas, em ordem decrescente de valor de importância (VI), amostradas na floresta estacional semidecidual da Fazenda Canchim, São Carlos, SP. N. = número de indivíduos; DA = densidade absoluta; DR = densidade relativa; $\mathrm{FA}=$ frequiência absoluta; $\mathrm{FR}=$ frequiência relativa; $\mathrm{DoA}=$ dominância absoluta (área basal); DoR = dominância relativa.

\begin{tabular}{|c|c|c|c|c|c|c|c|c|}
\hline Espécie & $\mathrm{N}$. & $\begin{array}{c}\text { DA } \\
\left(\text { ind.ha }^{-1}\right)\end{array}$ & $\begin{array}{l}\mathrm{DR} \\
(\%)\end{array}$ & $\begin{array}{l}\text { FA } \\
(\%)\end{array}$ & $\begin{array}{l}\text { FR } \\
(\%)\end{array}$ & $\begin{array}{c}\text { DoA } \\
\left(\mathrm{m}^{2} \cdot \mathrm{ha}^{-1}\right)\end{array}$ & $\begin{array}{r}\text { DoR } \\
(\%)\end{array}$ & $\begin{array}{l}\mathrm{VI} \\
(\%)\end{array}$ \\
\hline Mansoa difficilis & 103 & 137,33 & 19,51 & 48,00 & 11,43 & 0,1529 & 14,44 & 45,38 \\
\hline Acacia aff. plumosa & 51 & 68,00 & 9,66 & 34,67 & 8,25 & 0,1376 & 13,00 & 30,91 \\
\hline Thynantus elegans & 14 & 18,67 & 2,65 & 9,33 & 2,22 & 0,0932 & 8,80 & 13,68 \\
\hline Clytostoma campanulatum & 25 & 33,33 & 4,73 & 26,67 & 6,35 & 0,0268 & 2,53 & 13,62 \\
\hline Heteropterys sp. & 25 & 33,33 & 4,73 & 14,67 & 3,49 & 0,0475 & 4,49 & 12,71 \\
\hline Forsteronia cf. pubescens & 29 & 38,67 & 5,49 & 17,33 & 4,13 & 0,0298 & 2,82 & 12,43 \\
\hline Stizophyllum perforatum & 23 & 30,67 & 4,36 & 21,33 & 5,08 & 0,0302 & 2,85 & 12,29 \\
\hline Melloa quadrivalvis & 12 & 16,00 & 2,27 & 10,67 & 2,54 & 0,0746 & 7,05 & 11,86 \\
\hline Stigmaphyllon lalandianum & 16 & 21,33 & 3,03 & 17,33 & 4,13 & 0,0453 & 4,28 & 11,44 \\
\hline Serjania laruotteana & 14 & 18,67 & 2,65 & 14,67 & 3,49 & 0,0388 & 3,67 & 9,81 \\
\hline Lundia obliqua & 19 & 25,33 & 3,60 & 18,67 & 4,45 & 0,0176 & 1,66 & 9,71 \\
\hline Adenocalymna bracteatum & 22 & 29,33 & 4,17 & 12,00 & 2,86 & 0,0255 & 2,41 & 9,43 \\
\hline Bauhinia aff. microstachya & 6 & 8,00 & 1,14 & 8,00 & 1,90 & 0,0615 & 5,81 & 8,85 \\
\hline Arrabidaea triplinervea & 15 & 20,00 & 2,84 & 13,33 & 3,17 & 0,0295 & 2,79 & 8,80 \\
\hline Pereskia aculeata & 14 & 18,67 & 2,65 & 14,67 & 3,49 & 0,0215 & 2,03 & 8,18 \\
\hline Amphilophium vauthieri & 12 & 16,00 & 2,27 & 13,33 & 3,17 & 0,0253 & 2,39 & 7,84 \\
\hline Friedericia speciosa & 14 & 18,67 & 2,65 & 10,67 & 2,54 & 0,0228 & 2,15 & 7,35 \\
\hline Urvillea laevis & 10 & 13,33 & 1,89 & 10,67 & 2,54 & 0,0166 & 1,57 & 6,00 \\
\hline Arrabidaea mutabilis & 10 & 13,33 & 1,89 & 5,33 & 1,27 & 0,0202 & 1,91 & 5,07 \\
\hline Pristimera andina & 7 & 9,33 & 1,33 & 9,33 & 2,22 & 0,0127 & 1,20 & 4,75 \\
\hline Thinouia mucronata & 7 & 9,33 & 1,33 & 9,33 & 2,22 & 0,0116 & 1,10 & 4,64 \\
\hline Davilla rugosa & 8 & 10,67 & 1,52 & 8,00 & 1,90 & 0,0087 & 0,82 & 4,24 \\
\hline Pithecoctenium crucigerum & 6 & 8,00 & 1,14 & 6,67 & 1,59 & 0,0120 & 1,13 & 3,86 \\
\hline Arrabidaea conjugata & 5 & 6,67 & 0,95 & 5,33 & 1,27 & 0,0110 & 1,04 & 3,26 \\
\hline Mikania sp. & 5 & 6,67 & 0,95 & 6,67 & 1,59 & 0,0036 & 0,34 & 2,88 \\
\hline Acacia aff. paniculata & 5 & 6,67 & 0,95 & 4,00 & 0,95 & 0,0100 & 0,94 & 2,84 \\
\hline Condylocarpon isthmium & 5 & 6,67 & 0,95 & 4,00 & 0,95 & 0,0064 & 0,60 & 2,50 \\
\hline Serjania caracasana & 5 & 6,67 & 0,95 & 4,00 & 0,95 & 0,0060 & 0,57 & 2,47 \\
\hline Semialarium paniculatum & 5 & 6,67 & 0,95 & 2,67 & 0,64 & 0,0092 & 0,87 & 2,45 \\
\hline Hippocratea volubilis & 4 & 5,33 & 0,76 & 5,33 & 1,27 & 0,0037 & 0,35 & 2,38 \\
\hline Dalbergia frutescens & 4 & 5,33 & 0,76 & 4,00 & 0,95 & 0,0055 & 0,52 & 2,23 \\
\hline Arrabidaea selloi & 3 & 4,00 & 0,57 & 4,00 & 0,95 & 0,0048 & 0,45 & 1,97 \\
\hline Macfadyena unguis cati & 3 & 4,00 & 0,57 & 2,67 & 0,64 & 0,0048 & 0,45 & 1,66 \\
\hline Heteropterys pauciflora & 3 & 4,00 & 0,57 & 2,67 & 0,64 & 0,0048 & 0,45 & 1,66 \\
\hline Tetrapterys guilleminiana & 2 & 2,67 & 0,38 & 2,67 & 0,64 & 0,0037 & 0,35 & 1,36 \\
\hline Pyrostegia venusta & 2 & 2,67 & 0,38 & 2,67 & 0,64 & 0,0033 & 0,31 & 1,33 \\
\hline Forsteronia $\mathrm{sp}$ & 2 & 2,67 & 0,38 & 2,67 & 0,64 & 0,0027 & 0,26 & 1,27 \\
\hline Machaerium dimorphandrum & 3 & 4,00 & 0,57 & 1,33 & 0,32 & 0,0031 & 0,29 & 1,18 \\
\hline Indeterminada 1 & 2 & 2,67 & 0,38 & 1,33 & 0,32 & 0,0050 & 0,47 & 1,17 \\
\hline Anemopaegma chamberlaynii & 2 & 2,67 & 0,38 & 2,67 & 0,64 & 0,0017 & 0,16 & 1,18 \\
\hline Trigonia sp. & 2 & 2,67 & 0,38 & 1,33 & 0,32 & 0,0020 & 0,19 & 0,88 \\
\hline Strychnos sp. & 1 & 1,33 & 0,19 & 1,33 & 0,32 & 0,0022 & 0,21 & 0,71 \\
\hline Paragonia pyramidata & 1 & 1,33 & 0,19 & 1,33 & 0,32 & 0,0012 & 0,11 & 0,62 \\
\hline Urvillea ulmacea & 1 & 1,33 & 0,19 & 1,33 & 0,32 & 0,0010 & 0,09 & 0,60 \\
\hline Tetrapterys chlorocarpa & 1 & 1,33 & 0,19 & 1,33 & 0,32 & 0,0007 & 0,07 & 0,57 \\
\hline Total & 528 & 704,00 & 100,00 & 420,00 & 100,00 & 1,0586 & 100,00 & 300,00 \\
\hline
\end{tabular}


determinação das associações formadas pelas lianas que atualmente encontram-se neste fragmento.

Agradecimentos - Ao CNPq pela bolsa concedida, à Embrapa/CPPSE, por colocar à disposição a infraestrutura da Fazenda; ao Programa de Pós Graduação em Ecologia da UFSCar (PPG/ERN). Aos especialistas da Unicamp e do Instituto de Botânica - SP, com quem tive contacto para identificação de parte do material botânico. A Genise Sommer, Júlio A. Lombardi e aos professores Marco A. Assis e Neusa T. Ranga, entre outros, também pela identificação das espécies e, em especial, às colegas Ana C. Kim e Andréia A. Rezende, pela imensa ajuda que prestaram.

\section{Referências bibliográficas}

ARANHA, C., BACCHI, O. \& LEITÃO FILHO, H.F. 1982. Plantas invasoras de culturas. v. 2. Instituto Campineiro de Ensino Agrícola, Campinas.

BERNACCI, L.C. \& LEITÃO FILHO, H.F. 1996. Flora fanerogâmica da floresta da Fazenda São Vicente, Campinas, SP. Revista Brasileira de Botânica 19:149-164.

CALDERANO FILHO, B., SANTOS, H.G., FONSECA, O.O.M., SANTOS, R.D., PRIMAVESI, O. \& PRIMAVESI, A.C. 1998. Os solos da Fazenda Canchim, Centro de Pesquisa de Pecuária do Sudeste, São Carlos, SP: levantamento semidetalhado, propriedades e potenciais. Embrapa-CPPSE, São Carlos.

CITADINI-ZANETTE, V., SOARES, J.J. \& MARTINELLO, C.M. 1997. Lianas de um remanescente florestal da microbacia do Rio Novo, Orleans, Santa Catarina, Brasil. Insula 26:45-63.

ENGEL, V.L., FONSECA, R.C.B. \& OLIVEIRA, R.E. 1998. Ecologia de lianas e o manejo de fragmentos florestais. Série Técnica Ipef 12:43-64.

GENTRY, A.H. 1982. Patterns of neotropical plant species diversity. Evolutionary Biology 15:1-84.

GENTRY, A.H. 1991. The distribuition and evolution of climbing plants. In The biology of vines, (F.E. Putz \& H.A. Mooney, eds.). Cambridge University Press, Cambridge, p.3-49.

HEGARTY, E.E. \& CABALLÉ, G. 1991. Distribuition and abundance of vines in forest communities. In The biology of vines (F.E. Putz \& H.A. Mooney, eds.). Cambridge University Press, Cambridge, p.313-336.

HORA, R.C. 1999. Composição florística e aspectos da estrutura da comunidade de lianas em uma mata mesófila semidecídua na Fazenda Canchim, São Carlos - SP. Dissertação de mestrado, Universidade Federal de São Carlos, São Carlos.

KIM, A.C. 1996. Lianas da mata atlântica do Estado de São Paulo. Dissertação de mestrado. Universidade Estadual de Campinas, Campinas.
LEITÃO FILHO, H.F. 1987. Considerações sobre a florística de florestas tropicais e sub-tropicais do Brasil. Série Técnica Ipef 35:41-46.

LIMA, H.C., LIMA, M.P.M., VAZ, A.M.S.F. \& PESSOA, S.V.A. 1997. Trepadeiras da reserva ecológica de Macaé de Cima. In Serra de Macaé de Cima: Diversidade florística e conservação em Mata Atlântica. (H.C. Lima \& R.R. Guedes-Bruni, eds.). Jardim Botânico do Rio de Janeiro, Rio de Janeiro, p.75-87.

MAGURRAM, A.E. 1988. Ecologycal diversity and its measurement. Princeton University Press, Princeton.

MAIA, L.M.A. 1990. Aspectos fitossociológicos de lianas em mata de terra firme, Manaus-Amazonas. Dissertação de mestrado, Instituto Nacional de Pesquisa Amazônica, Manaus.

MORELLATO, L.P.C. \& LEITÃO FILHO, H.F. 1996. Reproductive phenology of climbers in a Southeastern Brazilian Forest. Biotropica 28:180-191.

MORELLATO, L.P.C. \& LEITÃO FILHO, H.F. 1998. Levantamento florístico da comunidade de trepadeiras de uma floresta semidecídua no sudeste do Brasil. Boletim do Museu Nacional 103:1-15.

MÜLLER-DOMBOIS, D. \& ELLEMBERG, H. 1974. Aims and methods of vegetation ecology. John Wiley \& Sons, New York.

OLIVEIRA FILHO, A.T. 1989. Composição florística e estrutura comunitária da floresta de galeria do córrego da Paciência, Cuiabá (MT). Acta Botanica Brasilica 3:91-112.

PEIXOTO, A.L. \& GENTRY A.H. 1990. Diversidade e composição florística da mata de tabuleiro na Reserva Florestal de Linhares (Espírito Santo, Brasil). Revista Brasileira de Botânica 13:19-25.

PIELOU, E.C. 1975. Ecological diversity. WileyInterscience, New York.

PUTZ, F.E. 1984. The natural history of lianas on Barro Colorado Island, Panama. Ecology 65:1713-1724.

PUTZ, F.E. 1990. Liana stem diameter growth and mortality rates on Barro Colorado Island, Panama. Biotropica 22:103-105.

REZENDE, A.A. 1997. Levantamento florístico das espécies de lianas da Estação Ecológica do Nordeste Paulista - São José do Rio Preto/Mirassol, SP, Chave de identificação e diagnoses. Dissertação de mestrado, Universidade Estadual de Campinas, Campinas.

TERAMURA, A.H., WARREN, G.G. \& FORSETH, I.N. 1991. Physiological ecology of mesic, temperate woody vines. In The biology of vines (F.E. Putz \& H.A. Mooney, eds.). Cambridge University Press, Cambridge, p.245-286.

TOLENTINO, M. 1967. Estudo crítico sobre o clima da região de São Carlos. Concurso de monografias municipais. Prefeitura Municipal de São Carlos, São Carlos.

VENTURI, S. 2000. Florística e fitossociologia do componente apoiante-escandente em uma floresta costeira subtropical. Dissertação de mestrado, Universidade Federal do Rio Grande do Sul, Porto Alegre. 